\title{
Anti-Glomerular Basement Membrane Glomerulonephritis in an HIV Positive Patient: Case Report
}

\author{
Eduardo José Bellotto Monteiro ${ }^{1}$, Daiane Caron', \\ Carlos Alberto Balda ${ }^{1}$, Marcello Franco ${ }^{2}$, \\ Aparecido Bernardo Pereira ${ }^{1}$ and \\ Gianna Mastroianni Kirsztajn ${ }^{1}$
}

\author{
${ }^{1}$ Nephrology Service/ Department of Medicine and ${ }^{2}$ Department \\ of Pathology, Federal University of São Paulo-Medical \\ School; São Paulo, SP, Brazil
}

\begin{abstract}
We report on a case of a patient with HIV infection, diagnosed 18 months prior to the development of an anti-glomerular basement membrane (anti-GBM) rapidly progressive glomerulonephritis; this is probably the first report of such an association. A 30-year-old white man presented with elevation of serum creatinine $(1.3-13.5 \mathrm{mg} / \mathrm{dL}$ within one month $)$. At admission, the urinalysis showed proteinuria of $7.2 \mathrm{~g} / \mathrm{L}$ and 8,000,000 erythrocytes/mL. Renal biopsy corresponded to a crescentic diffuse proliferative glomerulonephritis mediated by anti-GBM, and serum testing for anti-GBM antibodies was positive; antinuclear antibodies (ANA) and anti-neutrophilic cytoplasmic antibodies (ANCA) were also positive. The patient underwent hemodyalisis and was treated with plasmapheresis, cyclophosphamide and prednisone. The association described here is not casual, as crescentic glomerulonephritis is not common in HIV-positive patients, anti-GBM glomerulonephritis is rare and anti-GBM antibodies are frequently observed in $\mathrm{HIV}$-positive subjects when compared to the overall population. Based on the current case and on the elevated frequency of the positivity for such antibodies in this group of patients, it is advisable to be aware of the eventual association between these two conditions and to promote an active search for anti-GBM antibodies and early diagnosis of eventual urinary abnormalities in HIV-positive subjects, considering the severity of anti-GBM glomerulonephritis.

Key Words: Anti-GBM glomerulonephritis, HIV, urinary abnormalities.
\end{abstract}

Goodpasture's syndrome includes glomerulonephritis, lung hemorrhage and glomerular basement membrane antibodies (GBM) [1]. Lung hemorrhage occurs in 50 to $70 \%$ of the cases, with greater incidence in smokers. It may be determined or exacerbated by pulmonary infection or hypervolemia and can also occur in the absence of significant renal disease [2,3]. From an epidemiological point of view, HLA-DR 15 seems to be the most important risk factor for the development of this disease. HLA-DR7 has also been related to the process, but it is considered to be less involved [4].

In the case of renal involvement, antibodies are strongly linked to the GBM in a linear pattern, and sometimes weakly to the Bowman's capsule and to the tubular basement membrane, as demonstrated by immunofluorescence. This technique is probably sensitive enough for identification of the antibodies, but it is not totally specific, as the linear pattern has already been demonstrated in kidney allografts, diabetes mellitus, systemic lupus erithematosus, fibrillary glomerulonephritis, and even in normal kidneys [2]. In addition, circulating anti-GBM autoantibodies can be demonstrated by several other available assays with different sensitivities and specificities [2,3].

Received on 18 November 2005; revised 13 January 2006.

Address for correspondence:Dr.Gianna Mastroianni Kirsztajn. Escola Paulista de Medicina - UNIFESP. Rua Botucatu, 740. Zip code: 04023-900 São Paulo-SP Brazil. Phone: + 551155746300. Fax + 5511 55739652.E-mail: giannamk@uol.com.br

The Brazilian Journal of Infectious Diseases 2006;10(1):55-58. (C) 2006 by The Brazilian Journal of Infectious Diseases and Contexto Publishing. All rights reserved.
Lung hemorrhage is not a constant finding, both in patients and in experimental animal models, and it has a weak correlation with the serum levels of anti-GBM antibodies. Initially, it was thought that the pulmonary and the renal antigens were distinct. Recently, it has been demonstrated that the antigen is the same in both tissues. However, the accessibility of the antibody to the antigen is different in the two tissues. While a highly specific antibody to the basement membrane will link to the GBM after an intravenous injection, probably due to the fenestrated nature of the glomerular endothelium, there should be previous insult in the alveolus that exposes the antigen epitopes in order to allow binding to circulating antibodies. Therefore, there seems to be an association between toxicity by oxygen, smoking, organic solvents, infection and the development of lung hemorrhage $[5,6]$. Recently, it has been suggested that the exposition of the antigen by tissue injury might be the main pathogenic mechanism [7].

We report on a case of a patient with HIV infection, diagnosed 18 months prior to the development of an antiGBM rapidly progressive glomerulonephritis. To the best of our knowledge, this is the first report of such an association.

\section{Case Report}

A 30-year-old white man presented with a history of acute hepatitis B virus 18 months previously, when the diagnosis of HIV infection was also established and a year previously, secondary syphilis was diagnosed. At hospitalization, he presented with nephritic syndrome and acute renal failure. The serum creatinine increased from 1.3 to $13.5 \mathrm{mg} / \mathrm{dL}$ in one 
month. At admission, the initial urinalysis showed proteinuria of $7.2 \mathrm{~g} / \mathrm{L}$ and $8,000,000$ erythrocytes $/ \mathrm{mL}$, with evident erythrocytic dismorfism and 30,000 leukocytes $/ \mathrm{mL}$. The patient had clinical and serological criteria of cured hepatitis B (negative $\mathrm{HbsAg}$, positive anti-HBc IgG, negative Hbe Ag, positive anti-HBs) and for syphilis (positive VDRL 1/4 decreasing titre, positive FTA-Abs). His initial blood count revealed hemoglobin $13.9 \mathrm{~g} / \mathrm{dL}$, hematocrit $40 \%$, leukocytes $7,100 / \mathrm{mL}$, platelets $260,000 / \mathrm{mL}$; a chest X-ray was normal; the physical examination revealed no alteration and his blood pressure was $120 \times 80 \mathrm{mmHg}$. Ultrasonography showed both kidneys of normal size. He was submitted to a renal biopsy. Light microscopy showed eight glomeruli with focal fibrinoid necrosis, substantial endocapillary hypercellularity, a moderate degree of neutrophilic infiltration and circumferential cellular crescents in 50\% of the sampled glomeruli; the intact capillary walls had normal thickness (Figure 1-3); in addition, there was moderate tubular acute necrosis; the immunofluorescence technique revealed linear fluorescent deposits of $\operatorname{IgG}$ and $\mathrm{C} 3$ along the GBM. In summary, the renal biopsy corresponded to a progressive diffuse proliferative glomerulonephritis mediated by anti-GBM. Serum testing for anti-GBM antibodies was positive, with a titer of $68 \mathrm{U} / \mathrm{mL}$; serum tests for ANA and ANCA were positive; total complement activity was $160 \mathrm{UCH} 50 / \mathrm{mL}$ (reference value: 130 to 330 ), $\mathrm{C} 298 \%$ (RV: $>70 \%$ ), C3 $116 \mathrm{mg} / \mathrm{dL}$ (RV: 90 to 180 ), C4 $27 \mathrm{mg} / \mathrm{dL}$ (RV: 10 to 40), negative for anti-dsDNA, -RNP, -Sm, -Ro and -La, undetectable HIV viral load and $\mathrm{CD}_{4} 495 / \mathrm{mL}$ (RV $>1,000)$. The patient underwent hemodyalisis and was treated with 14 sessions of plasmapheresis, a low dose of cyclophosphamide $(1.5 \mathrm{mg} / \mathrm{kg})$ and prednisone $(1 \mathrm{mg} / \mathrm{kg})$ for two weeks. The treatment was discontinued due to the persistence of signs and symptoms and development of leukopenia. The patient was maintained in dialysis and did not present any infectious complications or pulmonary symptomatology within a six-month follow-up period.

\section{Discussion}

HIV-positive patients may present with renal involvement related to the infection itself, to supervening infections or to the side effect of drugs used in treatment $[8,9]$.

Several glomerulopathies have been observed in HIVpositive patients, such as IgA nephropathy, membranous glomerulonephritis, proliferative glomerulonephritis, minimal change disease, and particularly the collapsing variant of focal segmental glomerulosclerosis, which is an usual presentation of HIV-associated nephropathy [10-14].

We present a case of anti-GBM rapidly progressive glomerulonephritis in an HIV positive patient, an association that, to the best of our knowledge, has not been previously reported.

For treatment, considering the severity of the renal failure, we decided to use the same immunosuppressive regimen recommended for patients with anti-GBM glomerulonephritis, as the viral load of the patient was undetectable and he did not present with other active or recent infections that would contraindicate such a procedure. This treatment is based on the premise that the autoantibodies are responsible for the tissular lesion, and the aim is their removal and blockage of their synthesis. Plasmapheresis (4L/day during 14 days) concomitant to the administration of cyclophosphamide (2-3 $\mathrm{mg} / \mathrm{kg} / \mathrm{day})$ and prednisone $(1 \mathrm{mg} / \mathrm{kg} /$ day $)$ for three months has been successfully used $[2,3,15]$. Post-treatment relapse is rare, and in some cases has been related to smoking. In nontreated cases, antibody titers can be detected in the blood for up to one year or more [3]. For those patients progressing to irreversible renal failure, renal transplantation can be performed safely after the antibodies are no longer detectable in the blood. Some researchers consider it wise to wait at least six months after the first post-therapy negative result or at least two years for non-treated patients [3].

When the treatment is initiated with serum creatinine inferior to $600 \mu \mathrm{mol} / \mathrm{L}(6.7 \mathrm{mg} / \mathrm{dL}), 80 \%$ to $90 \%$ of the patients are maintained without the need for dialysis after one year. When treatment is initiated with the patients already in dialysis or with serum creatinine superior to $600 \mu \mathrm{mol} / \mathrm{L}$, this percentage falls to $0 \%$ to $18 \%$ [2]. Alternatively, immunoadsorption with protein A has been used in substitution for plasmapheresis $[16,17]$. Also, mycophenolate mofetil was administered with success to one patient with frequent relapses of lung hemorrhage, utilizing the normal dosage [18].

In our patient, there was no evidence of a short-term response, and as the patient developed leukopenia with the use of cyclophosphamide, after conclusion of the conventional initial treatment, the immunosuppressive drugs were discontinued.

The most intriguing aspect of this case report is the association between anti-GBM glomerulonephritis and HIV infection. Currently, there is sufficient evidence that HIV is etiopathogenetically involved in several autoimmune phenomena [19]. Antibodies against platelets and white blood cells have been demonstrated in patients with AIDS [20]. It has also been demonstrated that T-cells infected by HIV may expose previously occult $\mathrm{CD}_{4}$ epitopes, leading to downregulation of the $\mathrm{CD}_{4}$ molecules, mediated by monoclonal antibodies against $\mathrm{CD}_{4}$ or gp-120 [21]; in addition, there is evidence that an autoimmune response mediated by T-cells is involved in the pathophysiology of HIV infection [21], and that it is likely that IL-6 and IL-10 are involved in the development of malignancy and autoimmunity in AIDS [22].

The association between membranous nephropathy and vasculitis with anti-GBM antibodies favors the hypothesis that GBM damage in these diseases can trigger an autoimmune response against GBM [2]. This hypothesis is also further corroborated by two case reports that describe patients who developed Goodpasture's syndrome after lithotripsy [23,24]. There is also an epidemiological association between exposure 
Figure 1. Diffuse crescentic glomerulonephritis, accompanied by acute tubular necrosis. Note granular casts in tubular lumens (PAS; $100 \mathrm{x}$ )

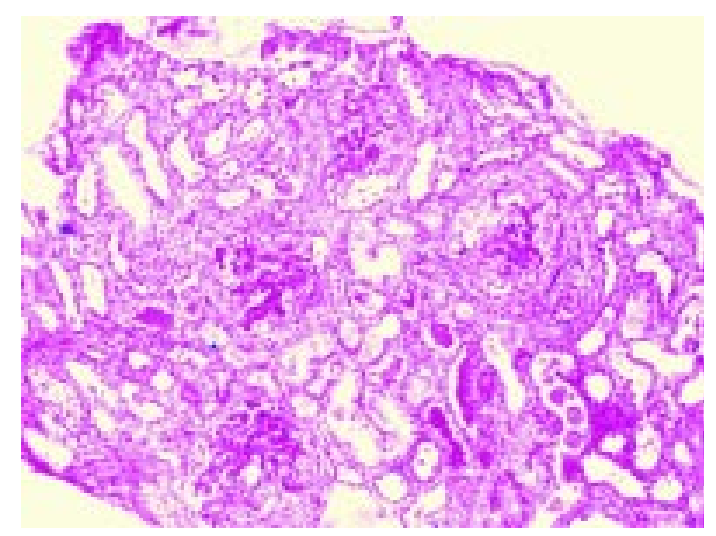

Figure 3. Linear immunofluorescence for $\operatorname{IgG}$ along the glomerular capillary wall (400x)

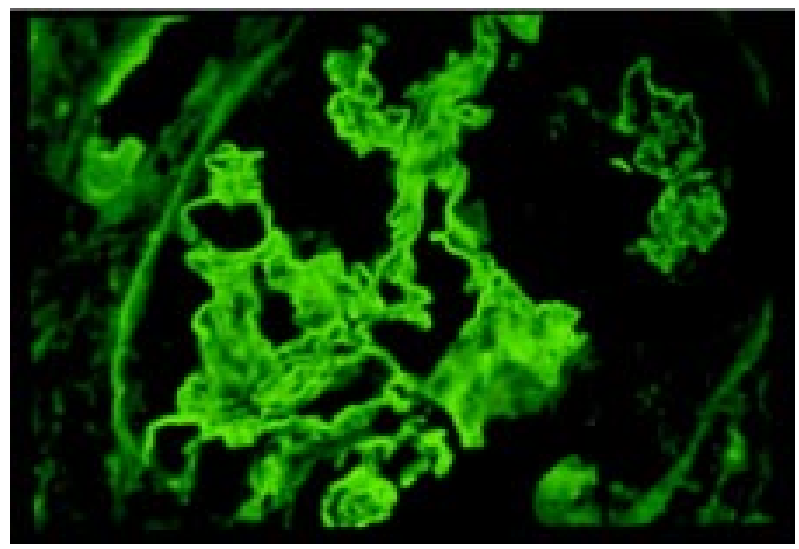

to organic solvents and progressive glomerulonephritis, with the eventual development of Goodpasture's syndrome [2]. The antigen involved is the $\alpha 3$ chain of type IV collagen, the main structural component of the glomerular basement membrane. The antigenic region of the molecule is the 230 amino acid non-collagen domain called $\mathrm{NC} 1$, in the carboxyl terminal region $[1,2,25]$.

Calderon et al. [1] detected anti-GBM antibodies, with evidence of anti-GBM glomerulonephritis, in HIV-negative patients with Pneumocystis carinii lung infection, and not in HIV-negative patients who carried the pathogen but did not have pneumonia, which suggested that the alveolar lesion caused by the agent is responsible for the development of the autoimmune disease. Moreover, HIV-positive patients with $P$. carinii pneumonia did not present anti-GBM antibodies.

On the other hand, Savige et al. [26] found anti-GBM antibodies in $17 \%$ of HIV-positive patients, with a significant correlation between the presence of the antibody and $\mathrm{CD}_{4}$ counts lower than 400/mL. Also, antinuclear antibodies were found in $23 \%$ and ANCA in $17 \%$ of the HIV-positive subjects. There was no correlation between the presence of antibodies
Figure 2. A large cellular crescent with a partially destroyed and compressed capillary tuft (PAS; 400x)

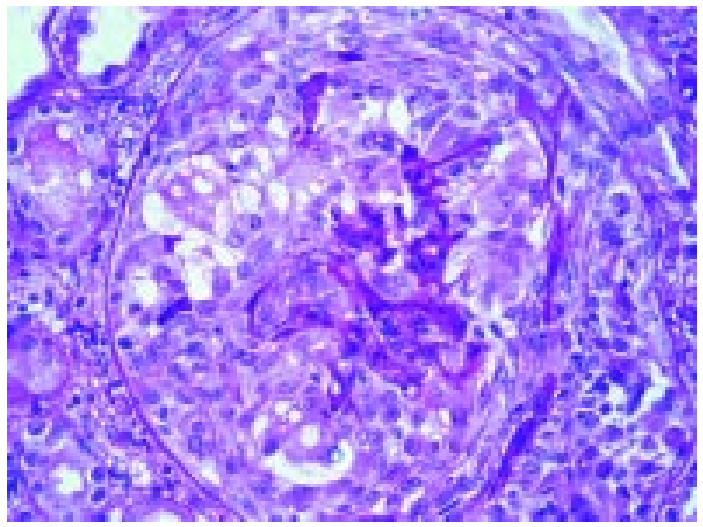

and the development of autoimmune disease. Even with moderate and elevated titers of anti-GBM antibodies in one of the cases, there was no clinical or laboratory evidence of glomerular disease. It is noteworthy that even normal individuals may have increased serum levels of some autoantibodies with no manifestation of disease [27-29].

Our report yields the hypothesis that the immune response in HIV patients may be etiopathogenetically involved in the development of anti-GBM glomerulonephritis. Some findings reinforce the idea that the association described in this case is not casual, such as: crescentic glomerulonephritis is not common in HIV-positive patients [26], anti-GBM glomerulonephritis is rare and anti-GBM antibodies are frequently observed in HIV-positive subjects when compared to the overall population [26].

We conclude that HIV-positive subjects with anti-GBM antibodies deserve special attention concerning the early diagnosis of eventual urinary abnormalities, considering the severity of anti-GBM glomerulonephritis. It is intriguing that anti-GBM glomerulonephritis has not been reported more frequently in HIV-patients, due to the number of these patients with anti-GBM antibodies in their serum and that these antibodies have a recognized pathogenetic role in such glomerulonephritis [26]. Based on our case report and on the elevated frequency of positivity for such antibodies in this group of patients, it is advisable to be aware of a possible association between these two conditions and to promote an active search for anti-GBM antibodies in HIV-positive subjects.

\section{References}

1. Calderon E.J., Wichmann I., Varela J.M., et al. Presence of glomerular basement membrane (GBM) antibodies in HIVpatients with Pneumocystis carinii pneumonia. Clin Exp Immunol 1997; 107:448-50.

2. Turner A.N., Rees A.J. Anti-glomerular basement membrane disease. In: Pusey C.D., Rees A.J. eds. Rapidly Progressive Glomerulonephritis. Oxford: Oxford University Press, 1998. 
3. Turner A.N. Goodpasture's disease. Nephrol Dial Transplant 2001;16:52-4.

4. Phelps R.G., Rees A.J. The HLA complex in Goodpasture's disease: a model for analyzing susceptibility to autoimmunity. Kidney Int 1999;56:1638-53.

5. Downie G.H., Roholt O.A., Jennings L., et.al. Experimental anti-alveolar basement membrane antibody-mediated pneumonitis. II. Role of endothelial damage and repair, induction of autologous phase, and kinetics of antibody deposition in Lewis rats. J Immunol 1982;129:2647-52.

6. Jennings L., Roholt O.A., Pressman D., et.al. Experimental antialveolar basement membrane antibody-mediated pneumonitis. I. The role of increased permeability of the alveolar capillary wall induced by oxygen. J Immunol 1981;127:129-34.

7. Kalluri R., Gattone V.H., Noelken M.E., Hudson B.G. The alpha 3 chain of type IV collagen induces autoimmune Goodpasture syndrome. Proc Natl Acad Sci U S A 1994;91:6201-5.

8. Weiner N.J., Goodman J.W., Kimmel P.L. The HIV-associated renal diseases: Current insight into pathogenesis and treatment. Kidney Int 2003;63(5):1618-31.

9. Karras A., Lafaurie M., Furco A., et.al. Tenofovir-related nephrotoxicity in human immunodeficiency virus-infected patients: three cases of renal failure, Fanconi syndrome, and nephrogenic diabetes insipidus. Clin Infect Dis 2003;36(8):1070-3.

10. Williams D.I., Williams D.J., Williams I.G., et.al. Presentation, pathology, and outcome of HIV associated renal disease in a specialist centre for HIV/AIDS. Sex Transm Infect 1998;74(3):179-84.

11. Bologna R.M. HIV-associated nephropathy: clinical characteristics and therapeutic options. AIDS Read 1999;9(1):38-42.

12. Kimmel P.L. Renal diseases in patients with HIV infection: a spectrum of outcomes in search of understanding. AIDS Read 1999;9(1):25-7.

13. Schwimmer J.A., Markowitz G.S., Valeri A., Appel G.B. Collapsing glomerulopathy. Semin Nephrol 2003;23(2):209-18.

14. Herman E.S., Klotman P.E. HIV-associated nephropathy: Epidemiology, pathogenesis, and treatment. Semin Nephrol 2003;23(2):200-8.

15. Glassock R.J. Crescentic glomerulonephritis. In: Ponticelli C., Glassock R.J. eds. Treatment of primary glomerulonephritis. Oxford: Oxford University Press, 1997.

16. Bygren P., Freiburghaus C., Lindholm T., et.al. Goodpasture's syndrome treated with staphylococcal protein A immunoadsorption. Lancet 1985;2:1295-6.
17. Laczika K., Knapp S., Derfler K., et.al. Immunoadsorption in Goodpasture's syndrome. Am J Kidney Dis 2000;36:392-5.

18. Garcia-Canton C., Toledo A., Palomar R. et.al. Goodpasture's syndrome treated with mycophenolate mofetil. Nephrol Dial Transplant 2000;15:920-2.

19. Morrow W.J., Isenberg D.A., Sobol R.E., et.al. AIDS virus infection and autoimmunity: a perspective of the clinical, immunological, and molecular origins of the autoallergic pathologies associated with HIV disease. Clin Immunol Immunopathol 1991;58:163-80.

20. van der Lelie J., Lange J.M., Vos J.J., et.al. Autoimmunity against blood cells in human immunodeficiency-virus (HIV) infection. Br J Haematol 1987;67:109-14.

21. Caporossi A.P., Bruno G., Salemi S., et.al. Autoimmune T-cell response to the CD4 molecule in HIV-infected patients. Viral Immunol 1998; 11:9-17.

22. Emilie D., Zou W., Fior R., et.al. Production and roles of IL-6, IL-10, and IL-13 in B-lymphocyte malignancies and in Blymphocyte hyperactivity of HIV infection and autoimmunity. Methods 1997;11:133-42.

23. Guerin V., Rabian C., Noel L.H. et.al. Anti-glomerularbasement-membrane disease after lithotripsy. Lancet 1990;335:856-7.

24. Umekawa T., Kohri K., Iguchi M. et.al. Glomerular-basementmembrane antibody and extracorporeal shock wave lithotripsy. Lancet 1993;341:556.

25. Wieslander J., Kataja M., Hudson B.G. Characterization of the human Goodpasture antigen. Clin Exp Immunol 1987;69:33240.

26. Savige J.A., Chang L., Horn S., Crowe S.M. Anti-nuclear, antineutrophil cytoplasmic and anti-glomerular basement membrane antibodies in HIV-infected individuals. Autoimmunity 1994;18:205-11.

27. Bilinska Z.T., Caforio A.L., Kusmierczyk-Droszcz B.K., et.al. Increased frequency of organ-specific cardiac antibodies in healthy relatives of patients with dilated cardiomyopathy: evidence for autoimmunity in Polish families. Clin Cardiol 1996;19(10):794-8.

28. Shimojo N., Katsuki T., Saito K., et.al. Thyroglobulin-specific T-cell line from a healthy individual does not produce proinflammatory cytokines on antigenic stimulation: an implication for possible fail-safe mechanism to avoid autoimmunity. Autoimmunity 1994;17(3):203-7.

29. Venables P.J., Rigby S., Mumford P.A., et.al. Autoimmunity to $\mathrm{La}$ (SS-B) in vitro is related to HLA-DR3 in healthy subjects. Ann Rheum Dis 1988;47(1):22-7. 